\title{
Generator Dynamic Performance Affected by Parameter's Uncertainty
}

\author{
${ }^{1}$ Akbar Beik Khormizi, ${ }^{2}$ Mohammad Reza Aghamohammadi and ${ }^{2}$ Mina Rezaee \\ ${ }^{1}$ School of Electrical and Computer Engineering, McMaster University, Ontario, Hamilton, Canada \\ ${ }^{2}$ Department of Electrical Engineering, Power and Water University of Technology, Tehran, Iran
}

Received 2012-10-31, Revised 2013-05-10; Accepted 2013-06-08

\begin{abstract}
This study investigates the effect of generator parameters inaccuracy on the transient stability performance of generator and power system. Normally, generator parameters are identified either by calculation based on design specification or by measurements. In both approaches the parameters are obtained with some degree of inaccuracy. Inaccuracy and uncertainty in the obtained parameters can affect the dynamic performance and transient behavior of synchronous generators in which this may affect transient stability evaluation of power systems. In this study by introducing a sensitivity analysis concept for dynamic performance of generator the effect of inaccuracy of generator parameters on the transient stability of power system is evaluated and the acceptable and tolerable inaccuracy in the identification of each parameter is analyzed. The proposed concept and approach is demonstrated on the IEEE 39-bus test.
\end{abstract}

Keywords: Parameter Identification, Parameter Inaccuracy, Parameter Uncertainty, Dynamic Performance, Generator Parameters, Transient Stability, Sensitivity Analysis

\section{INTRODUCTION}

Knowledge of the operational parameter of generators is necessary for performing stability studies and post mortem analysis of power systems. Traditionally, synchronous machine parameters are evaluated by off-line tests using the traditional methods for modeling synchronous machines (IEEE Guide, 2009). The procedures carried out in these methods usually involve difficult and time-consuming tests, such as ShortCircuit, Standstill Frequency Response (SSFR) and Open Circuit Frequency Response (OCFR) tests (Grune et al., 2012; Sellschopp and Arjona, 2007). In fact in these approaches parameters are determined individually and when they are used collectively to simulate a synchronous generator there are errors. To overcome the drawbacks of off-line methods, identification methods based on on-line measurements have been proposed (Boileau et al., 2011; Karrari and Malik, 2004). In all methods of modeling and parameter identification of synchronous generator, inaccuracy and error in the parameters identified for generators can affect generators dynamic performance that may affect system stability. Therefore, to accurately model, simulate and analyze the generator and the power system, it is essential to study the effect of parameter inaccuracy on performance of the system.

In this study the effect of the error and inaccuracy, which may exist for the identified parameters of the generators, on the dynamic performance and stability of generators and power system is investigated. Transient stability is adopted as criterion for dynamic performance of synchronous machines and power system. Moreover, the amount of inaccuracy in generator parameters that could dominantly affect its dynamic performance and stability is evaluated.

\subsection{Generator Model Accuracy}

Fundamental equations of synchronous machine were derived by Park and others years ago (Krause et al., 2003). Park's equations have the simplest form and Ontario, Hamilton, Canada 
are most well-known. In park's model, three-phase windings $\mathrm{a}, \mathrm{b}$ and $\mathrm{c}$ are replaced by two-phase windings, $d$ on the $d$-axis and $q$ on the $q$-axis. There are two damper windings $\mathrm{D}$ on the d-axis and $\mathrm{Q}$ on the q-axis. Damper windings are permanently shortcircuited. There is also a field winding $F$ on the d-axis and is dc-excited. During the development of synchronous machine theory many reactances and time constants have been defined. These parameters that are regarded as generator parameters include synchronous reactance for steady-state analysis, transient and subtransient reactances which include the effect of field winding and damper winding for transient analysis and other dynamic studies and the transient and subtransient time constants associated with reactance. All resistances are relatively small compared to the associated reactance. Generator parameters are provided either by manufacturers or by identification methods. The accuracy of generator modeling mainly depends upon the accuracy of generators parameters. For this purpose, many techniques and methodologies have been developed for identification of generator parameters. Different identification approaches could result in different degree of accuracy for parameters which could result in different dynamic behavior of generators and power system. Nevertheless, the question is how accurate the parameters of synchronous generators should be estimated to be able to provide a relatively accurate model for generators. In other words, in the process of parameters identification how much deviation in the obtained value for each parameter could be regarded as permissible accuracy. This study tries to answer these questions.

In order to evaluate the permissible inaccuracy for generator parameters, in this study, the dynamic performance of generators is taken as criteria for evaluating permissible deviation of each parameter. Following a fault and during transient stability of power systems the sensitivity of the first swing of each generator with respect to each parameter is considered as an index for stability performance of the generator. The smaller the magnitude of the first swing the more stable the generator and the greater the margin of generator stability. The tolerable change caused in the magnitude of the first swing of rotor angle due to the change in the parameters determines the permissible deviation in the parameter as the tolerable inaccuracy for that parameter. By ranking the sensitivity of the first swing with respect to the parameters the most dominating and crucial parameters for generator stability performance are recognized.

\subsection{Sensitivity Analysis of Generator Dynamic Behavior}

Sensitivity of the first swing of rotor angle oscillation with respect to parameters is taken as an index for evaluating the effect of parameter changes on the transient stability performance of generators. The first swing of rotor angle oscillation is a complex and nonlinear function of generator parameters. In order to evaluate the sensitivity of first swing since there is no analytic functionality between first swing and parameters, its sensitivity with respect to generator parameters is calculated numerically by time domain simulation. The procedure adopted in this study for evaluating sensitivity is as follows.

The initial values of generator parameters that may be obtained either from manufacturer or by identification are considered as base values. First swing oscillation of each generator following a threephase short circuit fault is also considered as corresponding performance of generators. For each generator an associated base first swing magnitude corresponding to the base parameters of the generators is calculated. Then, each dynamic parameter of a selected generator is changed individually from its base value by the step size of $10 \%$ until $\pm 30 \%$ change while other parameters are kept constant. Then by applying each individual change to the selected generator and performing transient stability simulation by DIgSILENT software first swing oscillation of the selected generator and other generators are calculated. Using deviation of the first swing oscillation corresponding to $\pm 30 \%$ changes of parameters the sensitivity of first swing with respect to each parameter of generator are calculated by Equation 1:

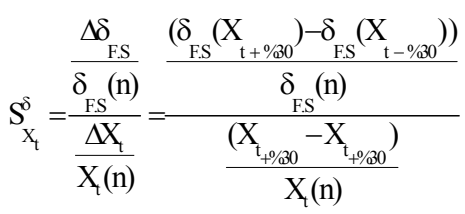

where, $\mathrm{S}_{\mathrm{x}_{\mathrm{t}}}^{\delta}$ is the sensitivity of generator first swing to parameter $X_{t} ; \delta_{F . S}$ is the magnitude of the first swing of the rotor oscillation; $X_{t}$ is the dynamic parameter of the generator; $X_{t}(n)$ is the base value of $\mathrm{X}_{\mathrm{t}} ; \delta_{\mathrm{F} . \mathrm{S}}(\mathrm{n})$ is the base value of the first swing of rotor 
angle; $X_{t-30 \%}$ is a $30 \%$ decrease in the value of $X_{t}$; $\mathrm{X}_{\mathrm{t}+30 \%}$ is a $30 \%$ increase in the value of $\mathrm{X}_{\mathrm{t}} ; \delta_{\mathrm{F} . \mathrm{S}}\left(\mathrm{X}_{\mathrm{t}}\right.$ $30 \%)$ is $\delta_{F . S}$ associated to $30 \%$ decrease in the value of $\mathrm{X}_{\mathrm{t}} ; \delta_{\mathrm{F} . \mathrm{S}}\left(\mathrm{X}_{\mathrm{t}-30 \%}\right)$ is $\delta_{\mathrm{F} . \mathrm{S}}$ associated to $30 \%$ increase in the value of $X_{t}$.

These sensitivities are considered as indices for finding the most dominating parameters affecting the stability performance of generators.

\subsection{Algorithm of Proposed Approach}

In order to demonstrate the proposed approach for finding the most dominating generator parameters on the stability performance of generators and power system IEEE 39-bus system consisting of two hydro, six steam and two Combined Cycle (CC) generators is adopted with the generator parameters taken from (Anderson and Fouad, 2003). In this study Generators no. 32, 34, 35 and 36 are selected for investigating the effect of their parameters on the stability performance.

Two kinds of studies are conducted (1) effect of changing the parameters of a specific generator on its own behavior and (2) effect of changing the parameters of other generators on dynamic behavior of a specific generator. Also for simulating transient stability behavior of generators and power system two types of faults are defined as (1) a three-phase short circuit fault on the terminal of the generator which is considered as severe fault and (2) a three-phase short circuit fault near to the terminal of the generator which is considered as medium fault. Moreover, for changing generator parameters two situations are considered (1) changing parameters without regarding consistency and (2) changing parameters with regarding consistency. The most important consistency relations between generator parameters that have been considered in this study are:

$X_{d}>X_{q}>X_{q}^{\prime}>X_{d}^{\prime}>X_{q}^{\prime \prime}>X_{d}^{\prime \prime}>T_{d}^{\prime}>T_{d}^{\prime \prime}$

\subsection{Effect of Changes Of Parameters of a Generator on its Own Dynamic Behavior}

Figure 1 illustrates the algorithm of effect of change of parameters of a generator on its own behavior. This algorithm is used for both sensitivity analyses without and with considering parameters consistency as stated in Equation 2. In the following discussion, two cases are discussed separately. In this fist case sensitivity analysis are studied without parameter consistency while in the second case parameter consistency is also taken into account.

\subsection{Sensitivity Analysis without Parameters Consistency}

In this case by changing each parameter of generators individually without regarding consistency of parameters and performing transient stability simulation for the applied fault the normalized sensitivity of the first swing of rotor angle with respect to each parameter of the generators for medium and severe fault is evaluated as shown in the Table 1 and 2 respectively.

It is worth noting that in order to better study the effect of each parameter on the stability performance of the generator and due to non-linear behavior of rotor angle the generator under test is operated close to the boundary of its stability. For this purpose the fault clearing time for severe fault is adopted close to the Critical Clearing Time (CCT) associated to the generator while the clearing time of medium fault is chosen as $0.1 \mathrm{sec}$. Because the hydro generator is salient pole then $X_{q}=X_{q}^{\prime}$, also $T_{q 0}^{\prime}$ is not definable so, in Table 1 and $2 X_{q}^{\prime}$ and $T_{q 0}^{\prime}$ have no values. As it can be seen from Table 1 showing the result of medium fault without consistency for generators no.35, 32 parameters $\mathrm{X}_{\mathrm{q}}, \mathrm{X}_{\mathrm{q}}^{\prime}$ and $\mathrm{H}$ while for generators no.34, 36 parameters $X_{q}, X_{q}^{\prime}$ and $H$ have the highest sensitivity which means these parameters have most dominating effect on the first swing and consequently on the transient stability performance of the generators. From Table 2 it is clear that parameters $X_{q}, X_{d}^{\prime}$ and $H$ for generators no.32, 35, 36 have the highest sensitivity while for generator no. $34 \mathrm{X}_{\mathrm{q}}, \mathrm{X}_{\mathrm{d}}^{\prime}, \mathrm{H}$ and $\mathrm{X}_{\mathrm{d}}^{\prime \prime}$ have the highest sensitivity. In the cases that the sensitivity of first swing rotor angle with respect to a parameter is positive it means that decrease of that parameter causes a decrease in the first swing which leads to more stability and when the associated sensitivity of a parameter is negative it means that increase of that parameter leads to more stability. As it can be seen from Table 1 and 2 for medium fault applied to generators no.32, 35 and 36 the sensitivity of the first swing rotor angle with respect to machine inertia $\mathrm{H}$ is negative while for generator no.34 this sensitivity is positive and in the case of severe fault for all generators this sensitivity is negative. 
Akbar Beik Khormizi et al. / American Journal of Applied Sciences 10 (6): 570-578, 2013

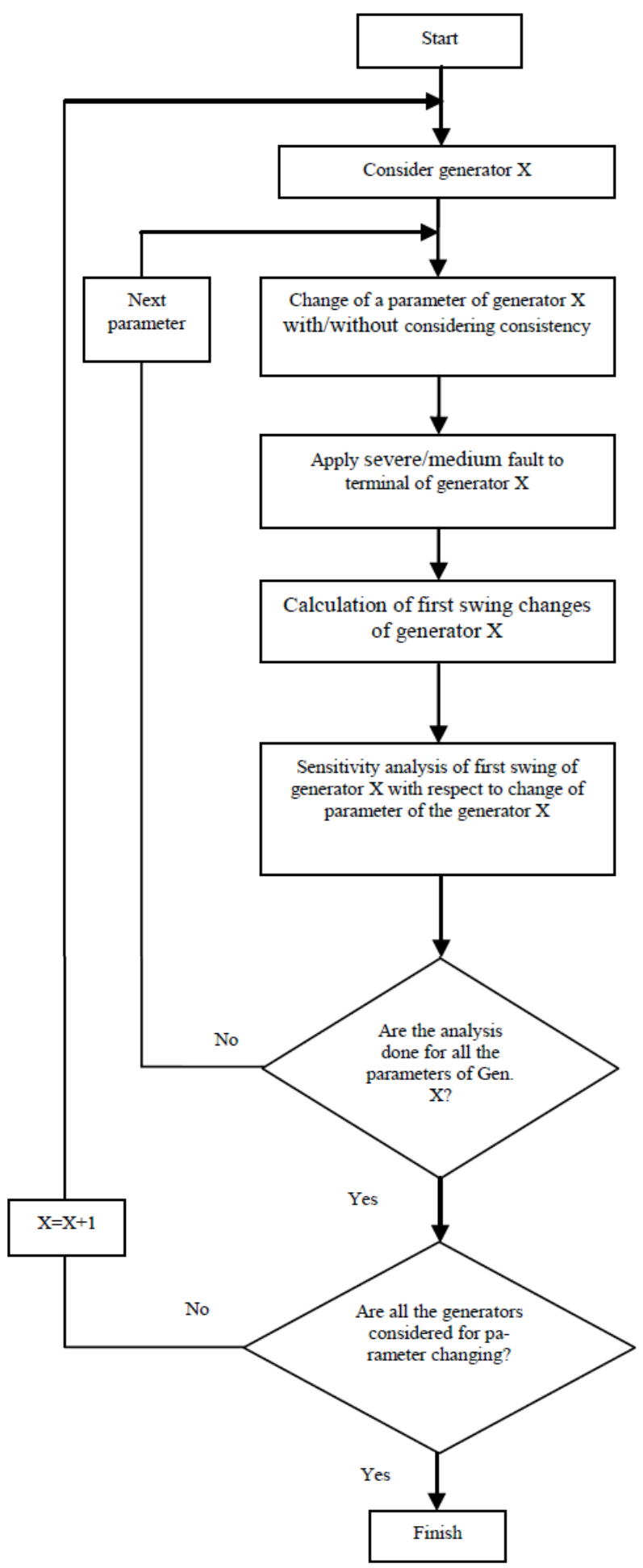

Fig. 1. Algorithm of effect of change of parameters of a generator on its own performance 
Akbar Beik Khormizi et al. / American Journal of Applied Sciences 10 (6): 570-578, 2013

Table 1. Sensitivity of the first swing of rotor angle of generators with respect to their parameters without consistency when medium fault is applied

\begin{tabular}{lccrr}
\hline Gen. No. & Hydro G35 & Steam G34 & Steam G32 & CC G36 \\
\hline MVA & 231.60 & 233.00 & 384.00 & 192.000 \\
Fault Location & Line22-21 & Line16-19 & Line14-13 & Line24-23.0 \\
Fault Duration & 0.10 & 0.10 & 0.10 & 0.1 .00 \\
Parameter Sensitivity Sensitivity Sensitivity Sensitivity & & & \\
$\mathrm{X}_{\mathrm{d}}$ & 0.0205 & -0.002 & 0.0160 & 0.0152 \\
$\mathrm{X}_{\mathrm{d}}^{\prime}$ & 0.3209 & -0.020 & 0.1720 & 0.0996 \\
$\mathrm{X}_{\mathrm{d}}^{\prime \prime}$ & -0.0793 & -0.018 & 0.0510 & -0.0396 \\
$\mathrm{X}_{\mathrm{q}}$ & 0.4231 & -0.370 & 0.9960 & 0.8417 \\
$\mathrm{X}_{\mathrm{q}}^{\prime}$ & 0.1590 & -0.114 & -0.1206 & 0.0698 \\
$\mathrm{X}_{\mathrm{q}}^{\prime \prime}$ & 0.0233 & -0.004 & 0.0990 & -0.0530 \\
$\mathrm{~T}_{\mathrm{d} 0}^{\prime}$ & -0.0391 & 0.006 & -0.0640 & 0.0146 \\
$\mathrm{~T}_{\mathrm{d} 0}^{\prime \prime}$ & 0.0074 & 0.005 & -0.0410 & -0.0527 \\
$\mathrm{~T}_{\mathrm{q} 0}^{\prime}$ & -0.0140 & 0.009 & 0.0178 & -0.5284 \\
$\mathrm{~T}_{\mathrm{q} 0}^{\prime \prime}$ & -0.0374 & -0.050 & 0.1140 & -1.1690 \\
$\mathrm{H}$ & -0.2967 & 0.786 & \\
\hline
\end{tabular}

Table 2. Sensitivity of the first swing of rotor angle of generators with respect to their parameters without consistency when severe fault is applied

\begin{tabular}{|c|c|c|c|c|}
\hline Gen. No. & Hydro G35 & Steam G34 & Steam G32 & CC G36 \\
\hline MVA & 231.60 & 233.00 & 384.00 & 192.00 \\
\hline Fault Location & Bus 25.00 & Bus 34.00 & Bus 32.00 & Bus 36.00 \\
\hline Fault Duration & 0.220 & 0.24 & 0.186 & 0.25 \\
\hline Parameter & Sensitivity & Sensitivity & Sensitivity & Sensitivity \\
\hline$X_{d}$ & 0.022 & 0.066 & 0.0230 & 0.030 \\
\hline$X_{d}^{\prime}$ & 0.508 & 0.433 & 0.2270 & 0.331 \\
\hline$X_{d}^{\prime \prime}$ & -0.144 & -0.391 & -0.0650 & -0.051 \\
\hline$X_{q}$ & 0.278 & 0.338 & 0.3990 & 0.342 \\
\hline $\mathrm{X}_{\mathrm{q}}^{\prime}$ & -0.017 & 0.009 & 0.0030 & \\
\hline $\mathrm{X}_{\mathrm{q}}^{\prime \prime}$ & 0.008 & 0.009 & 0.0001 & 0.013 \\
\hline $\mathrm{T}_{\mathrm{d} 0}^{\prime}$ & -0.044 & -0.217 & -0.0940 & -0.112 \\
\hline $\mathrm{T}_{\mathrm{d} 0}^{\prime \prime}$ & 0.001 & -0.058 & -0.0460 & 0.014 \\
\hline $\mathrm{T}_{\mathrm{q} 0}^{\prime}$ & -0.007 & -0.002 & -0.0030 & \\
\hline $\mathrm{T}_{\mathrm{q} 0}^{\prime \prime}$ & 0.000 & 0.047 & 0.0160 & 0.016 \\
\hline $\mathrm{H}$ & -1.610 & -1.427 & -1.7430 & -1.484 \\
\hline
\end{tabular}

\subsection{Sensitivity Analysis with Parameters Consistency}

By changing each parameter of generators no.32, 34, 35 and 36 individually with considering parameters consistency and performing transient stability simulation for the medium and severe faults the sensitivity of first swing of the generators with respect to parameters are calculated. Table 3 and 4 show associated normalized sensitivities of the first swing with respect to each parameter of generators. By taking the consistency of parameters into account the preference of generator parameters ranked based on the sensitivity of first swing of rotor angle are shown in Table 5. For the generators under study there is a relation between their inertia as Equation 3:

$\mathrm{H}_{\mathrm{G} 34}>\mathrm{H}_{\mathrm{G} 36}=\mathrm{H}_{\mathrm{G} 35}>\mathrm{H}_{\mathrm{G} 32}$ 
Akbar Beik Khormizi et al. / American Journal of Applied Sciences 10 (6): 570-578, 2013

Table 3. Sensitivity of the first swing of rotor angle with respect to their parameters with consistency when medium fault is applied

\begin{tabular}{lccrr}
\hline Gen. No. & Hydro G35 & Steam G34 & Steam G32 & CC G36 \\
\hline MVA & 231.60 & 233.00 & 384.00 & 192.00 \\
Fault Location & Line22-21 & Line16-19 & Line14-13 & Line24-23 \\
Fault Duration & 0.10 & 0.10 & 0.10 & 0.10 \\
Parameter & Sensitivity & Sensitivity & Sensitivity & Sensitivity \\
$X_{d}$ & 0.1121 & -0.206 & 0.6010 & 0.4971 \\
$X_{d}^{\prime}$ & 0.2763 & -0.021 & 0.1730 & 0.0432 \\
$X_{d}^{\prime \prime}$ & -0.0715 & -0.022 & 0.1800 & 0.0310 \\
$X_{q}$ & 0.4231 & -0.371 & 0.6030 & 0.8047 \\
$X_{q}^{\prime}$ & 0.1590 & -0.114 & -0.1206 & 0.0310 \\
$X_{q}^{\prime \prime}$ & -0.0192 & -0.022 & 0.1500 & -0.0530 \\
$T_{d 0}^{\prime}$ & -0.0391 & 0.006 & -0.0640 & 0.0146 \\
$T_{d 0}^{\prime \prime}$ & 0.0074 & 0.005 & -0.0410 & -0.0527 \\
$T_{q 0}^{\prime}$ & -0.0140 & 0.009 & 0.0178 & -0.5284 \\
$T_{\mathrm{q} 0}^{\prime \prime}$ & -0.0374 & -0.050 & 0.1140 & -1.1690 \\
$\mathrm{H}^{\prime}$ & -0.2967 & 0.786 & & - \\
\hline
\end{tabular}

Table 4. Sensitivity of the first swing of rotor angle with respect to their parameters with consistency when severe fault is applied

\begin{tabular}{|c|c|c|c|c|}
\hline Gen. No. & Hydro G35 & Steam G34 & Steam G32 & CC G36 \\
\hline MVA & 231.60 & 233.00 & 384.00 & 192.00 \\
\hline Fault Location & Bus 25.00 & Bus 34.00 & Bus 32.00 & Bus 36.00 \\
\hline Fault Duration & 0.220 & 0.24 & 0.186 & 0.25 \\
\hline Parameter & Sensitivity & Sensitivity & Sensitivity & Sensitivity \\
\hline$X_{d}$ & 0.069 & 0.248 & 0.254 & 0.978 \\
\hline$X_{d}^{\prime}$ & 0.433 & 1.911 & 0.183 & 0.247 \\
\hline $\mathrm{X}_{\mathrm{d}}^{\prime \prime}$ & -0.141 & -0.380 & -0.030 & -0.038 \\
\hline$X_{q}$ & 0.278 & 0.235 & 0.408 & 0.353 \\
\hline$X_{q}^{\prime}$ & -0.017 & 0.009 & 0.003 & \\
\hline $\mathrm{X}_{\mathrm{q}}^{\prime \prime}$ & -0.075 & 0.008 & -0.037 & -0.038 \\
\hline $\mathrm{T}_{\mathrm{d} 0}^{\prime}$ & -0.044 & -0.217 & -0.094 & -0.112 \\
\hline $\mathrm{T}_{\mathrm{d} 0}^{\prime \prime}$ & 0.001 & -0.058 & -0.046 & 0.014 \\
\hline $\mathrm{T}_{\mathrm{q} 0}^{\prime}$ & -0.007 & -0.002 & -0.003 & \\
\hline $\mathrm{T}_{\mathrm{q} 0}^{\prime \prime}$ & 0.000 & 0.047 & 0.016 & 0.016 \\
\hline $\mathrm{H}$ & -1.610 & -1.427 & -1.743 & -1.484 \\
\hline
\end{tabular}

If sensitivity of first swing rotor angle of $i^{\text {th }}$ machine with respect to its inertia is noted as " $\mathrm{SG}_{\mathrm{i}}$ " it is seen from Table 1-4 that there is a relationship between the sensitivity of fist swings with respect to
$\mathrm{H}$ in both cases of consistency and not consistency for medium fault as follows Equation 4:

$\mathrm{S}_{\mathrm{G} 32}>\mathrm{S}_{\mathrm{G} 34}>\mathrm{S}_{\mathrm{G} 36}>\mathrm{S}_{\mathrm{G} 35}$ 
Akbar Beik Khormizi et al. / American Journal of Applied Sciences 10 (6): 570-578, 2013

Table 5. Preference of parameters of generators with considering consistency

\begin{tabular}{|c|c|c|c|c|c|c|c|}
\hline \multicolumn{4}{|c|}{ Fault near to generator terminal } & \multicolumn{4}{|c|}{ Fault on generator terminal } \\
\hline Hydro G35 & Steam G34 & Steam G32 & CC G36 & Hydro G35 & Steam G34 & Steam G32 & CC G36 \\
\hline $\mathrm{X}_{\mathrm{q}}$ & $\mathrm{H}$ & $\mathrm{H}$ & $\mathrm{X}_{\mathrm{q}}$ & $\mathrm{H}$ & $\mathrm{H}$ & $\mathrm{H}$ & $\mathrm{H}$ \\
\hline $\mathrm{H}$ & $\mathrm{X}_{\mathrm{q}}$ & $X_{\mathrm{q}}$ & $\mathrm{H}$ & $X_{d}^{\prime}$ & $\mathrm{H}$ & $\mathrm{X}_{\mathrm{q}}$ & $X_{d}$ \\
\hline$X_{d}^{\prime}$ & $X_{d}$ & $X_{d}$ & $\mathrm{X}_{\mathrm{q}}$ & $\mathrm{X}_{\mathrm{q}}$ & $\mathrm{X}_{\mathrm{d}}^{\prime \prime}$ & $X_{d}$ & $\mathrm{X}_{\mathrm{q}}$ \\
\hline$X_{d}$ & $X_{q}^{\prime}$ & $X_{d}^{\prime \prime}$ & $\mathrm{X}_{\mathrm{q}}^{\prime}$ & $\mathrm{X}_{\mathrm{d}}^{\prime \prime}$ & $X_{d}$ & $\mathrm{X}_{\mathrm{d}}^{\prime}$ & $X_{d}^{\prime}$ \\
\hline $\mathrm{X}_{\mathrm{d}}^{\prime \prime}$ & $\mathrm{T}_{\mathrm{d} 0}^{\mathrm{m}}$ & $X_{d}^{\prime}$ & $\mathrm{T}_{\mathrm{d} 0}^{\prime}$ & $X_{q}^{\prime \prime}$ & $\mathrm{X}_{\mathrm{q}}$ & $\mathrm{T}_{\mathrm{d} 0}^{\prime}$ & $\mathrm{T}_{\mathrm{d} 0}^{\prime}$ \\
\hline $\mathrm{T}_{\mathrm{d} 0}^{\prime}$ & $X_{d}^{\prime \prime}, X_{q}^{\prime \prime}$ & $\mathrm{X}_{\mathrm{q}}^{\prime \prime}$ & $\mathrm{T}_{\mathrm{q} 0}^{\prime \prime}$ & $X_{d}$ & $\mathrm{~T}_{\mathrm{d} 0}^{\prime}$ & $\mathrm{T}_{\mathrm{d} 0}^{\prime \prime}$ & $X_{d}^{\prime \prime}$ \\
\hline $\mathrm{T}_{\mathrm{q} 0}^{\prime \prime}$ & $X_{d}^{\prime}$ & $\mathrm{T}_{\mathrm{q} 0}^{\prime \prime}$ & $X_{d}^{\prime}$ & $\mathrm{T}_{\mathrm{d} 0}^{\prime}$ & $\mathrm{T}_{\mathrm{d} 0}^{\prime \prime}$ & $\mathrm{X}_{\mathrm{q}}^{\prime \prime}$ & $\mathrm{X}_{\mathrm{q}}^{\prime \prime}$ \\
\hline $\mathrm{X}_{\mathrm{q}}^{\prime \prime}$ & $\mathrm{T}_{\mathrm{q} 0}^{\prime}$ & $X_{q}^{\prime}$ & $\mathrm{X}_{\mathrm{d}}^{\prime \prime}$ & $\mathrm{T}_{\mathrm{d} 0}^{\prime \prime}$ & $\mathrm{T}_{\mathrm{q} 0}^{\prime \prime}$ & $\mathrm{X}_{\mathrm{d}}^{\prime \prime}$ & $\mathrm{T}_{\mathrm{q} 0}^{\prime \prime}$ \\
\hline $\mathrm{T}_{\mathrm{d} 0}^{\prime \prime}$ & $\mathrm{T}_{\mathrm{d} 0}^{\prime}$ & $\mathrm{T}_{\mathrm{d} 0}^{\prime}$ & $\mathrm{X}_{\mathrm{q}}^{\prime \prime}$ & $\mathrm{T}_{\mathrm{q} 0}^{\mathrm{m}}$ & $\mathrm{X}_{\mathrm{q}}^{\prime}$ & $\mathrm{T}_{\mathrm{q} 0}^{\prime \prime}$ & $\mathrm{T}_{\mathrm{d} 0}^{\prime \prime}$ \\
\hline- & $\mathrm{T}_{\mathrm{d} 0}^{\prime \prime}$ & $\mathrm{T}_{\mathrm{d} 0}^{\prime \prime}$ & $\mathrm{T}_{\mathrm{q} 0}^{\prime}$ & - & $\mathrm{X}_{\mathrm{q}}^{\prime \prime}$ & $\mathrm{X}_{\mathrm{q}}^{\prime}$ & $\mathrm{X}_{\mathrm{q}}^{\prime}$ \\
\hline- & - & $\mathrm{T}_{\mathrm{q} 0}^{\prime}$ & $\mathrm{T}_{\mathrm{d} 0}^{\prime \prime}$ & - & $\mathrm{T}_{\mathrm{q} 0}^{\prime}$ & $\mathrm{T}_{\mathrm{q} 0}^{\prime}$ & $\mathrm{T}_{\mathrm{q} 0}^{\prime}$ \\
\hline
\end{tabular}

Table 6. Effect of changing the parameters of other generators on dynamic behavior of generator no. 35

\begin{tabular}{lcccc}
\hline Paramete & $\frac{\mathrm{d} \delta_{\mathrm{F} . \mathrm{S}}(35)}{\mathrm{dX}(32)}$ & $\frac{\mathrm{d} \delta_{\mathrm{F} . \mathrm{S}}(35)}{\mathrm{d} \mathrm{X}_{\mathrm{t}}(34)}$ & $\frac{\mathrm{d} \delta_{\mathrm{F} . \mathrm{S}}(35)}{\mathrm{dX}(35)}$ & $\frac{\mathrm{d} \delta_{\mathrm{F} . \mathrm{S}}(35)}{\mathrm{dX}(36)}$ \\
\hline $\mathrm{X}_{\mathrm{d}}$ & 0.00004 & 0.00002 & 0.02200 & 0.011 \\
$\mathrm{X}_{\mathrm{d}}^{\prime}$ & -0.00010 & -0.00020 & 0.50800 & 0.039 \\
$\mathrm{X}_{\mathrm{d}}^{\prime \prime}$ & -0.00700 & -0.00490 & -0.1440 & -0.015 \\
$\mathrm{X}_{\mathrm{q}}$ & -0.00280 & 0.00050 & 0.27800 & 0.028 \\
$\mathrm{~T}_{\mathrm{q} 0}^{\prime \prime}$ & -0.00510 & -0.00770 & - & 0.012 \\
$\mathrm{X}_{\mathrm{q}}^{\prime \prime}$ & -0.00490 & -0.00500 & 0.00800 & -0.017 \\
$\mathrm{~T}_{\mathrm{d} 0}^{\prime}$ & -0.00010 & -0.00010 & -0.0440 & -0.006 \\
$\mathrm{~T}_{\mathrm{d} 0}^{\prime \prime}$ & 0.00020 & 0.00020 & 0.00800 & 0.007 \\
$\mathrm{X}_{\mathrm{q}}^{\prime}$ & 0.00040 & 0.00040 & - & -0.013 \\
$\mathrm{~T}_{\mathrm{q} 0}^{\prime \prime}$ & 0.00690 & 0.00670 & -0.0004 & 0.022 \\
\hline
\end{tabular}

While for the severe fault it is as follows Equation 5:

$$
\mathrm{S}_{\mathrm{G} 32}>\mathrm{S}_{\mathrm{G} 35}>\mathrm{S}_{\mathrm{G} 36}>\mathrm{S}_{\mathrm{G} 34}
$$

As it is clear from relations 4 and 5 for all cases first swing of generator no.32 with the smallest inertia has the highest sensitivity with respect to its inertia. It can be concluded that inaccuracy in the inertia of the generator with the smallest inertia could cause high error on the dynamic behavior of generator and system. Simulation results for both cases of consistency and not consistency show that although the generator no. 32 and 34 are steam units but because of having different MVA rating they have different sensitivities with respect to their parameters and therefore they have different dynamic behavior after a disturbance. Therefore, it can be concluded that in addition to the type of parameters, generator rating and severity of disturbance could change the effectiveness of parameter on the dynamic behavior of generators.

\subsection{Effect of the Parameters of a GENERATOR on Dynamic Behavior of Other Generators}

Algorithm of this approach is illustrated in Fig. 2. In this approach effect of changing the parameters of generators no.32, 34 and 36 on dynamic behavior of generator no.35 is investigated. 
Akbar Beik Khormizi et al. / American Journal of Applied Sciences 10 (6): 570-578, 2013

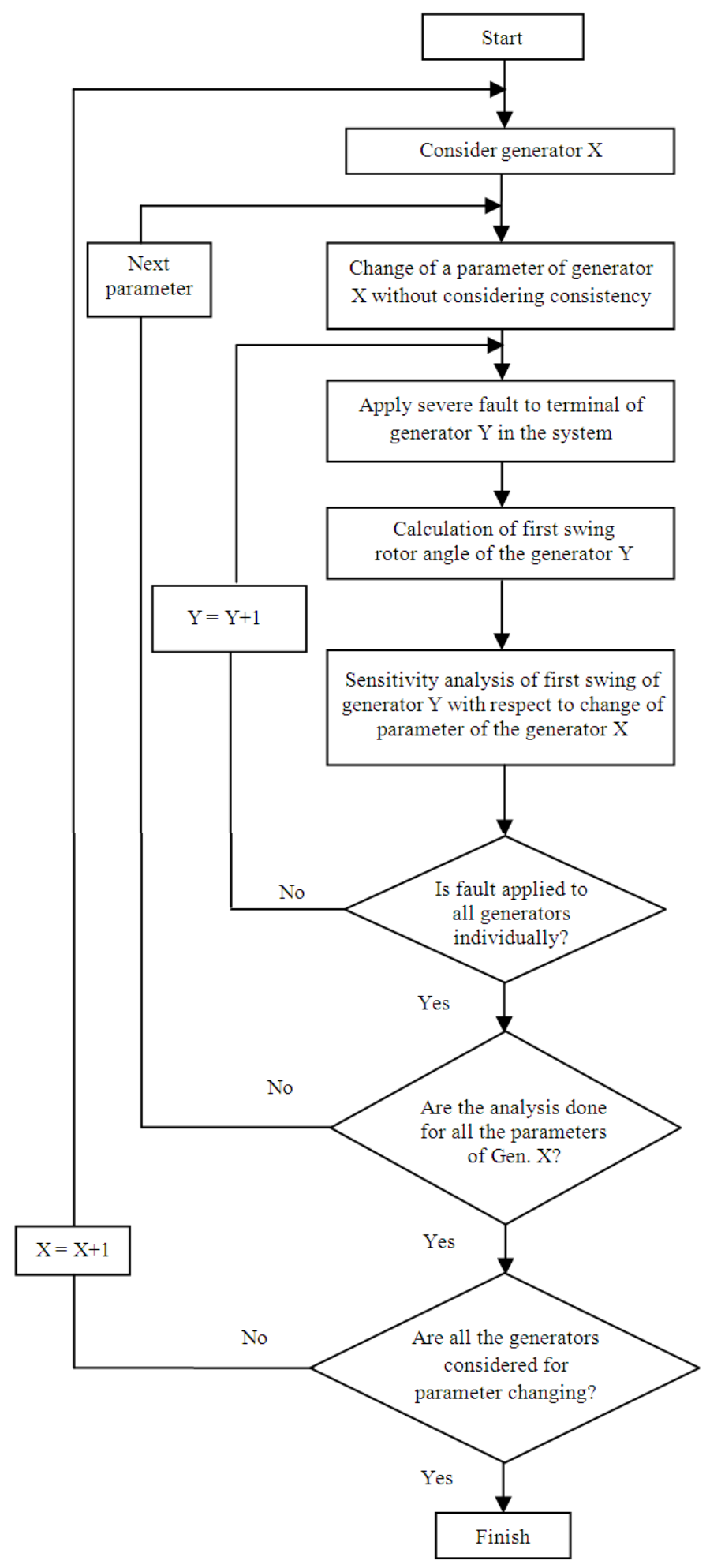

Fig. 2. Algorithm of effect of change of parameters of a generator on behavior of other generators 
In this investigation the following steps are taken. (1) changing a parameter of generator no.32. (2) applying severe fault on generator no.35. (3) calculating first swing rotor angle of generator no.35. (4) calculating sensitivity of first swing of generator no. 35 with respect to the changed parameter of generator no.32. (5) repeat the process from step 2 for another parameter of generator no.32. (6) repeat process from step 1 for generators no.34 and 36. By applying these steps, the results of sensitivities are summarized in Table 6. As it can be seen from Table 6, the sensitivity of generator no. 35 with respect to its own parameters is higher than its sensitivity with respect to other generators parameters. Therefore, dynamic behavior of a generator is more affected by inaccuracy in its own parameter in comparison with inaccuracy in the parameters of other generators. In addition, it can be seen that, the parameter $\mathrm{H}$ of other generators has more effect compared with other parameters of those generators, so, inaccuracy in identification of this parameter is more important rather than other parameters.

\section{CONCLUSION}

In this study, the effect of the inaccuracy of generator parameters on the dynamic behavior of that generator, other generators and power system has been considered. Transient stability and first swing oscillation of rotor angle is the dynamic behavior considered in this study. The sensitivity of the amplitude of the first swing rotor oscillation with respect to the parameters is considered as an index for evaluating the effectiveness of the parameters on the transient stability performance of generators and power system. This sensitivity is then used as a criterion for preferring the parameters of generators in affecting dynamic behavior of generators. Two scenarios for changing the parameters including consistency and not consistency are considered. In this study, two kinds of faults including medium fault and severe fault are considered.

Simulation results show that parameters $\mathrm{H}$, $X_{q}, X_{d}^{\prime}, X_{d}^{\prime \prime}, X_{d}$ have the most effect on the stability performance of generators, so, their inaccuracy could cause most error in the evaluation of generator behavior. Therefore, in identification of these parameters most care should be taken. However, for all cases inaccuracy in identification of parameter $\mathrm{H}$ has the most effect on dynamic behavior of generator and power system.

\section{REFERENCES}

Anderson, P.M. and A.A. Fouad, 2003. Power System Control and Stability. 2nd Edn., IEEE Press, Piscataway (N.J.), ISBN-10: 0471238627, pp: 658.

Boileau, T., N. Leboeuf, B. Nahid-Mobarakeh and F. Meibody-Tabar, 2011. Online identification of PMSM parameters: Parameter identifiability and estimator comparative study. IEEE Trans. Indus. Applic., $\quad 47$ : 1944-1957. DOI: 10.1109/TIA.2011.2155010

Grune, R., H. Einfeld and U. Schafer, 2012. Standstill frequency response tests for model identification of a synchronous machine. Proceedings of the 19th International Conference on Electrical Machines, Sept. 6-8, IEEE Xplore Press, Rome, pp: 1-6. DOI: 10.1109/ICELMACH.2010.5608076

IEEE Guide, 2009. 115-2009-IEEE Guide: Test procedures for synchronous machines part I-acceptance and performance testing part ii-test procedures and parameter determination for dynamic analysis. IEEE Xplore Digital Library. DOI: 10.1109/IEEESTD.2010.5464495

Karrari, M. and O.P. Malik, 2004. Identification of heffron-phillips model parameters for synchronous generators using online measurements. IEE Proc. Gener. Transm. Distrib., 151: 313-320. DOI: 10.1049/ip-gtd:20040275

Krause, P.C., O. Wasynczuk and S.D. Sudhoff, 2003. Analysis of Electric Machinery and Drive Systems. 2nd Edn., IEEE Press, New York, ISBN-10: 047114326X, pp: 613.

Sellschopp, F.S. and M.A. Arjona, 2007. Determination of synchronous machine parameters using standstill frequency response tests at different excitation levels. Proceeidngs of the IEEE International Electric Machines and Drives Conference, May 3-5, IEEE Xplore Press, Antalya, pp: 1014-1019. DOI: 10.1109/IEMDC.2007.382815 